\title{
Enfoque temático de enseñanza de habilidades: importancia de la competencia percibida
}

\author{
Gurutze LUIS-DE COS \\ Saioa URRUTIA-GUTIERREZ \\ Izaskun LUIS-DE COS
}

Datos de contacto:

Gurutze Luis-de Cos

Universidad de Antofagasta gurutze.luisdecos@uantof.cl

Saioa Urrutia-Gutierrez Universidad del País Vasco saioa.urrutia@ehu.eus

Izaskun Luis-de Cos Universidad del País Vasco Izaskun.luis@ehu.eus

\section{RESUMEN}

El enfoque temático de enseñanza de habilidades tiene como objetivo principal el desarrollo de la competencia motriz, constructo que en interacción con la competencia motriz percibida influye en el establecimiento y afianzamiento de hábitos de práctica físico-deportiva. El objetivo de este estudio es, por un lado, comprobar la relación entre la competencia percibida, la competencia motriz y la práctica físico-deportiva, y por el otro, analizar la precisión con la que evalúan los practicantes y no practicantes de actividad físico deportiva sus habilidades motrices. Los resultados confirman la relación positiva entre las tres variables y además, que los practicantes de actividad físico-deportiva, a pesar de sobreestimar su capacidad, son más precisos en sus valoraciones que los no practicantes. Este trabajo propone aplicar el enfoque temático de la enseñanza de habilidades en el ámbito formativo dada la importancia que tiene el desarrollo de la competencia motriz en la competencia percibida y por ende en el aumento de la práctica físico-deportiva, y se propone trabajarla.

PALABRAS CLAVE: Enfoque temático de enseñanza de habilidades; competencia motriz; Competencia percibida; práctica físico-deportiva. 


\title{
Skill theme approach: The importance of perceived of competence
}

\begin{abstract}
The purpose of the skill theme approach is the development of motor competence, a construct that in interaction with perceived motor competence influences the establishment and strenghtening of physical and sport activity habits. The aim of this study is on one hand to verify the relation between the perceived motor competence, motor competence and physical-sports practice and on the other, analyze his accuracy of perceived motor competence in practitioners and non-practitioners of physicalsport activity. The results confirm the positive relationship between the three variables and also that physical and sport activity practitioners, despite overestimating their ability, are more accurate in their assessments than non-practitioners. This work proposes to apply the skill theme approach due to the importance that motor competence has on perceived motor competence and therefore in increasing physical and sports practice.
\end{abstract}

KEYWORDS: Skill theme approach; motor competence; perceived motor competence; physical and sport practice.

\section{Introducción}

Los modelos de enseñanza en educación física son propuestas pedagógicas que buscan el desarrollo integral y consecución de competencias de niños y niñas. Sin embargo, cada modelo tiene sus peculiaridades. Así como algunos modelos elaboran propuestas metodológicas que se pueden aplicar a diferentes contenidos (modelo cooperativo, modelo de responsabilidad personal y social...) en otros modelos, el contenido da sentido al mismo. Este es el caso del Enfoque Temático de la Enseñanza de las Habilidades (ETEH) (Contreras, Arribas-Galarraga, y Gutiérrez, 2017).

Un modelo pedagógico, más allá de exponer el marco teórico de actuación basado en el conocimiento científico, facilita al docente de forma precisa su rol y el de su alumnado, el procedimiento concreto a seguir para la presentación del contenido, dirección de clase, estrategias de enseñanzaaprendizaje, así como orientación sobre el sistema de evaluación más adecuado para la verificación de las competencias a adquirir. Basándose en las teorías del desarrollo y aprendizaje motor, el ETEH diseña una progresión fija para el desarrollo y la adquisición de habilidades motrices, en la que la individualización es una premisa importante. Esta progresión comienza con el trabajo de habilidades básicas cuidadas, para después pasar a la 
combinación de estás habilidades y terminar con la integración progresiva de habilidades básicas en contextos de actividades físico-deportivas.

El ETEH es un modelo que se plantea adecuado para su desarrollo durante las etapas de Educación Infantil y Educación Primaria, pues es durante esos años, debido a los procesos madurativos y de desarrollo motor, cuando resulta más sencilla y efectiva la interiorización y mejora de las diferentes habilidades y destrezas. Precisamente el hecho de respetar las etapas madurativas hace que este modelo, a diferencia de los modelos tradicionales de educación física, se presente como una propuesta longitudinal, es decir, no propone una temporalización acotada a una edad y curso concreto, sino que para adaptarse al desarrollo del alumnado requiere una programación para al menos el año escolar (Contreras et al., 2017), siendo recomendable que se platee para cada ciclo escolar. No obstante, el refinamiento de las habilidades básicas es un proceso a largo plazo que deberia continuarse en las etapas de Educación Secundaria, más aún, a lo largo de toda la vida.

En definitiva, mediante este modelo se busca la creación de una base adecuada de competencia motriz, lo que permitirá al alumnado vivir experiencias de éxito que repercutirán positivamente en la competencia motriz percibida (Wang y Bian, 2013), es decir, en la creencia que cada individuo tenga sobre su capacidad para resolver diferentes situaciones motrices (Arruza y cols., 2011; Harter, 1978, AUTOCITA, 2014).

En esta linea, diversas investigaciones (Barnett, Dawes y Wilmut, 2012; Barnett, Morgan, van Beurden, y Beard, 2008; Barnett, van Beurden, Morgan, Brooks y Beard, 2009; Garcia, Rodriguez y Perez, 2013; Haugen, Ommundsen, y Seiler,2013; Inchley, Kirby, y Currie, 2011; Khodaverdi, Bahram, Khalaji, y Kazemnejad, 2013; Khodaverdi, Bahram, Stodden, y Kazemnejad, 2016; McIntyre, 2009; Lopes, Rodrigues, Maia, y Malina, 2011;Rigoli, Piek y Kane, 2011; Stodden, Gao, Goodway, y Langendorfer, 2014; Stodden, Langendorfer, y Roberton, 2009; Stooden y cols., 2008; Stodden, True, Langendorfer y Gao, 2013; Vedul-Kjelsås, Sigmundsson, Stensdotter, y Haga, 2012; Viholainen, Aro, Purtsi, Tolvanen, y Cantell, 2013) han analizado la relación entre la competencia motriz y la de competencia motriz percibida, y su influencia en los hábitos físico-deportivos, considerando a ambos elementos impulsores de la práctica físico-deportiva.

En un reciente estudio (Viholainen, Aro, Purtsi, Tolvanen, y Cantell 2013), presentaron un modelo conceptual en el que afirman, de acuerdo con autores como Harter $(1978,1988)$ y Eccles y Wigfield (2002), que la competencia motriz percibida influye en el mantenimiento o abandono de la práctica físicodeportiva.

Sin embargo, a diferencia de Harter, Eccles y Wigfield, Stodden y cols. (2008), Viholainen, Aro, Purtsi, Tolvanen, y Cantell (2013) sugieren que el concepto de competencia motriz es un mecanismo subyacente que interactúa con la competencia percibida, ejerciendo gran influencia en el mantenimiento y persistencia de la práctica físico-deportiva, por tanto, un elemento clave en 
la adherencia de la misma. A su entender, la competencia motriz percibida interviene y media a lo largo del desarrollo en la relación entre la competencia motriz y la práctica físico deportiva, siendo un proceso dinámico que se ve influenciado por el desarrollo del niño. De este modo, la competencia motriz del niño se modifica, originando cambios en la competencia motriz percibida y repercutiendo en la adhesión que puedan tener por la práctica fisicodeportiva.

Durante la etapa de escolarización de los niños y niñas se producen importantes cambios físicos y debido a estos cambios, pueden producirse desajustes en las valoraciones que hacen de sus propias habilidades. En ocasiones, las valoraciones erróneas pueden provocar consecuencias psicológicas que actúan como limitantes a la hora de iniciarse en ciertas actividades o incluso afectar a la capacidad de socializar con sus iguales. Es por ello, que autores como Raudsepp y Liblik (2002), Horn y Weiss (1991) y Weiss y Amorose (2005), resaltan la importancia de indagar en la diferencia que puede existir entre el nivel actual (real) de competencia motriz y la percepción que tienen de esa competencia, es decir en la precisión con la que los niños realizan las valoraciones sobre sus habilidades.

En esta línea, Weiss y Amorose (2005) llevaron a cabo una investigación con una muestra de niños y niñas de 8 a 14 años. En este estudio, analizaron el nivel de competencia motriz, de competencia percibida y la precisión con la que se percibian los jóvenes presentando tres niveles de precisión: sobreestimadores (se perciben más competentes de lo que son), subestimadores (se perciben menos competentes de lo que son) y precisos (se perciben tan competentes como lo son realmente).

Los estudios sobre la precisión de la percepción han mostrado que tanto el sexo como la edad influyen en la precisión con la que el alumnado valora su competencia (Dunn y Watkinson, 1994; Goodway y Rudisill, 1997; Sporis y cols., 2011; AUTOCITA, 2014; Washburn y Kolen, 2018; Weiss y Amorose, 2005). En cuanto al sexo, a pesar de la imprecisión tanto de chicos como de chicas en la valoración de sus habilidades, existen diferencia entre ellos y ellas a la hora de percibirse. De esta manera, mientras las chicas tienden a subestimar sus capacidades, los chicos sobrevaloran su nivel de competencia motriz.

En cuanto a la edad, los estudios analizados muestran que, a edades más tempranas, se tiende a sobreestimar las propias habilidades. A medida que aumenta la edad la competencia motriz percibida de los niños y las niñas disminuye y se muestran más exactos en sus valoraciones. Esto, según diversos autores (Harter, 1978; Horn y Weiss, 1991; Kim, 2003; Robinson, 2010; Weiss y Amorose, 2005) es debido al desarrollo cognitivo que se produce durante la maduración, que les permite ser más precisos en el análisis de su nivel de competencia motriz y diferenciar entre el esfuerzo, la suerte y la habilidad.

En virtud de lo anteriormente expuesto, existe suficiente evidencia científica que avala el interés de trabajar las habilidades y destrezas 
durante las etapas de educación infantil y primaria. Sin embargo, son escasos los estudios que abordan el resultado del trabajo realizado durante las citadas etapas. El presente estudio pretende poner en valor la importancia del modelo de enfoque temático de enseñanza de habilidades analizando la competencia y la competencia percibida en la etapa de la adolescencia (Educación Secundaria), pues se ha considerado que, tras terminar el periodo más sensible en el desarrollo de habilidades, es el momento idóneo para analizarlas. Por otro lado, la etapa de la adolescencia y juventud, viene siendo considerada, como apuntan diferentes autores (Balaguer, 2002; AUTOCITA, 2017,) una etapa fundamental en la adquisición de hábitos de vida que se mantendrán en la edad adulta. Con el objetivo de confirmar la relación entre la competencia motriz, la competencia percibida y los hábitos físico-deportivos de los jóvenes, en este estudio, se aborda la relación entre estas variables. Por otro lado, dada la importancia que tiene la precisión de la percepción de competencia en la confianza hacia determinadas tareas y actividades fisico-deportivas, se ha considerado importante conocer la precisión con la que valoran su nivel de competencia motriz los y las practicantes y no practicantes de actividades físico-deportivas.

\section{Método}

Los participantes en este estudio son 377 sujetos (182 Chicos y 195 chicas) que cursaban Educación Secundaria Obligatoria en 7 centros diferentes sitos los tres Territorios Históricos de la Comunidad Autónoma del País Vasco CAPV (Araba, Bizkaia y Gipuzkoa).

La muestra fue seleccionada por conveniencia, tomando como criterio de selección la disponibilidad e interés mostrado por los diferentes centros y profesores. Además, para este estudio se tuvieron en cuenta sólo se utilizó el modelo ETEH.

Diseño

El presente estudio es quasi-experimental de diseño "ex post facto" y naturaleza descriptiva, valorativa, correlacional y multivariada.

Variables de investigación

A continuación, se exponen las variables analizadas en esta investigación:

1. Competencia motriz: es la habilidad que tiene la persona para hacer frente a un problema motriz que se le presenta (real). Para obtener la variable de competencia motriz, se tipificaron los valores de las diez pruebas del test Sportcomp. Aquellas pruebas que se median en tiempo (7 metros pata coja, 7 metros pies juntos, desplazamiento sobre soportes y carrera ida y vuelta) se multiplicaron por -1, puesto que la valoración de estas pruebas era inversa al resto de pruebas, es decir, a mayor tiempo en estas pruebas, peor puntuación.

Después de tipificar los valores, por un lado, se sumaron los valores de las 
cinco pruebas de coordinación y por otro, se sumaron las cinco pruebas de condición. De esta manera, se realizó la media los dos bloques, obteniendo dos variables (capacidad coordinativa y capacidad condicional) con valores positivos y dos decimales, comprendidos entre 33 y 66 .

Para la obtención de la variable de competencia motriz, se sumaron los valores obtenidos en las dos variables de capacidades coordinativas y condicionales, y se realizó la media, obteniendo un valor que se sitúa entre 33 у 66.

2. Competencia motriz percibida: es la creencia que tiene cada individuo de su capacidad motora.

Para obtener este índice se realizó la suma de los valores obtenidos en los ítems que representaban la percepción de competencia motriz y después se tipifico el valor obtenido.

3. Práctica físico-deportiva: Se preguntó al alumnado si realizaban actividad fisico-deportiva.

Instrumentos

Adaptación de la Batería Sportcomp de Ruiz y cols., 2010 (Arruza, 2011)

La batería Sportcomp fue desarrollada por Ruiz y cols. en 2010, para medir y analizar el nivel de competencia motriz en adolescentes de $1^{\circ}$ a $4^{\circ}$ de la Educación Secundaria Obligatoria (ESO). La adaptación de dicha bateria resulto en dos variables: coordinación y control motor (saltos laterales, 7 metros saltando pies juntos, 7 metros saltando pata coja, desplazamiento sobre soportes y equilibrio) y aptitud fisica (Dinamometria, lanzamiento de balón medicinal, flexibilidad, carrera ida y vuelta, y abdominales).

Adaptación española del Test de Motivación de logro para el aprendizaje en Educación Física (Ruiz, Graupera y Rico, 2004)

Este cuestionario fue diseñado por Tamotsu Nishida para evaluar la motivación de logro para el aprendizaje en educación física. Más tarde Ruiz, Graupera, Gutierrez y Nishida (2004b), realizaron una adaptación de este cuestionario al castellano que consta de 37 items. En este estudio se aplicó una versión reducida de la versión española del Test AMPET que consta de 33 items y se tradujo al euskera.

Los análisis de fiabilidad de esta versión reducida en castellano muestran un nivel de fiabilidad de $\alpha=.81$. Los tres factores de esta escala muestran los siguientes coeficientes de consistencia interna: compromiso y seriedad $\alpha=$ .81 , compentecia motriz percibida $\alpha=.92 \mathrm{y}$ ansiedad ante el error y situaciones de estrés $\alpha=.79$.

La fiabilidad de la versión en euskera muestra un nivel aceptable de fiabiliadad, con un $\alpha=$.83. La consistencia interna para cada factor fue de $\alpha=$ .85 para la competencia motriz percibida, $\alpha=.68$ para el compromiso y seriedad y de alpha $=0.77$ para ansiedad ante el error y situaciones de estrés. Procedimiento de recogida de datos

Después de concretar los criterios de selección de la muestra, se realizó un primer contacto con los centros escolares de los tres TTHH, tomando como premisa que fuesen centros que impartieran los cursos desde $1^{\circ}$ a $4^{\circ}$ de la 
ESO. A la hora de establecer el contacto, se envió un informe explicativo detallando todos los pormenores del proyecto, o bien se concretó una cita con los responsables de la asignatura Educación Física, para explicar con detalle los objetivos y procedimientos del proyecto y la toma de datos.

A continuación, se pidieron los permisos pertinentes tanto al centro escolar como a los padres, madres y/o tutores responsables de los menores. Tras realizar el contacto y verificar la participación del centro, se acordó el calendario para la recogida de datos que duró 4 meses.

Para la aplicación de las pruebas y su organización, se establecieron bloques en función del tiempo disponible y la organización interna de cada centro. Si la sesión era de 1 hora, se pasaban las pruebas en 2 sesiones; si eran de 2 horas, en una sola sesión.

\section{Resultados}

En la tabla 1 se exponen las correlaciones entre la competencia motriz, la competencia percibida y la práctica físico-deportiva (correlación de Pearson). Como se puede observar, se refleja una relación positiva y significativa entre las tres variables $(\mathrm{p}<.01)$

Tabla 1

Correlaciones entre competencia motriz, competencia motriz percibida y práctica físicodeportiva

\section{CMP Práctica}

Competencia Motriz (CM)

Competencia Motriz Percibida (CMP)

Práctica físico-deportiva (Práctica)
1

$.341^{* *}$

1
$.215^{* *}$

$.314^{* *}$

Para analizar la relación entre la competencia percibida y competencia motriz en practicantes y no practicantes de actividad fisico-deportiva, se realizó una la comparación de medias de (tabla 2).

Los resultados indican que los jóvenes que practican algún tipo de actividad fisico-deportiva, presentan puntuaciones medias más altas en cuanto a la competencia que en la competencia motriz real. En cuanto al grupo de quienes no practican actividad fisico-deportiva alguna, se observan puntuaciones medias más altas en la competencia motriz real que en la percepción que tienen de su propia competencia. Las diferencias son estadísticamente significativas $(p<.000)$ y se producen entre ambas variables; 
en el caso de los practicantes de actividad fisico-deportiva, a favor de la competencia percibida, es decir, no son precisos en sus valoraciones y sobreestiman sus habilidades; en el caso de los no practicantes de actividad físico-deportiva, a favor de la competencia motriz, es decir, no son precisos en sus valoraciones y subestiman su capacidad.

Tabla 2

Diferencia de medias de la Competencia Motriz y la Competencia Motriz Percibida en función de la práctica

\begin{tabular}{ccccccccccc}
\hline & & \multicolumn{3}{c}{$\begin{array}{c}\text { Competencia } \\
\text { motriz }\end{array}$} & \multicolumn{4}{c}{$\begin{array}{c}\text { Competencia } \\
\text { percibida }\end{array}$} & $t$ & P \\
\cline { 2 - 7 } & $\mathbf{N}$ & $\mathbf{M}$ & $\mathbf{D T}$ & $\mathbf{N}$ & $\mathbf{M}$ & $\mathbf{D T}$ & & \\
\hline $\begin{array}{c}\text { Precisión de } \\
\text { percepción } \\
\text { de }\end{array}$ & Practicantes & 360 & 51,87 & 5,34 & 360 & 54,63 & 8,07 & - & 5.711 & $<.01$ \\
$\begin{array}{c}\text { competencia } \\
\text { motriz }\end{array}$ & $\begin{array}{c}\text { No } \\
\text { practicantes }\end{array}$ & 17 & 51,89 & 4,44 & 17 & 46,04 & 9,65 & 2,372 & $<.000$ \\
\hline
\end{tabular}

\section{Discusión y conclusiones}

Partiendo del supuesto de que el modelo del Enfoque Temático de la Enseñanza de las Habilidades (ETEH) se deberia desarrollar durante las etapas más sensibles del desarrollo motor puesto que, como indican Wang y Bian (2013), dotar al alumnado de vivencias positivas le permitirá adquirir experiencias de éxito que repercutirán positivamente en su competencia percibida, el presente estudio se ha centrado en analizar la competencia motriz, la competencia percibida y la práctica físico-deportiva y la relación entre ellas, en un grupo de jóvenes de la CAPV.

Tal y como afirman Stooden y cols. (2008), la competencia motriz podría ser precursora de la competencia percibida y que esto a su vez influiría en la práctica de actividad físico deportiva. De los resultados analizados, y siguiendo la tendencia de estudios anteriores, (Barnett y cols., 2012; Barnett y cols., 2008; Barnett y cols., 2009; Garcia y cols., 2013; Haugen y cols., 2013; Inchley, Kirby, y Currie, 2011; Khodaverdi y cols., 2013; Khodaverdi y cols., 2016; McIntyre, 2009; Lopes y cols., 2011;Rigoli y cols., 2011; Stodden y cols., 2014; Stodden, y cols., 2009; Stooden y cols., 2008; Stodden y cols., 2013; Vedul-Kjelsås y cols., 2012; Viholainen y cols., 2013; Wang y cols., 2013) se puede establecer una relación positiva y significativa entre la competencia motriz, la competencia percibida y la práctica físico-deportiva; a mayor competencia motriz y percepción de competencia, mayor práctica 
fisico-deportiva. Sin embargo, y a pesar de que Stodden y cols. (2008) y Lima y cols. (2017) describen la relación entre la competencia motriz y la práctica físico-deportiva como una relación recíproca, en este estudio no se puede determinar la causalidad entre las dos variables. No obstante, coincidimos con Wang y Bian (2013) en su reflexión que argumenta que realizar actividades fisico-deportivas que supongan un reto para el alumno, aumentará el tiempo dedicado a la práctica, desarrollando así nuevas habilidades y estrategias de respuesta a los diferentes problemas motrices, mejorando la percepción que tengan de las mismas. Asimismo, se sostiene que poseer un mayor nivel de competencia y un repertorio más amplio de respuestas motrices, favorecerá la competencia motriz percibida, lo que a su vez fomentará la participación en actividades y tareas motrices, y más específicamente en actividades de mayor complejidad.

Finalmente, en cuanto al análisis e interpretación de los datos obtenidos en relación a la práctica físico-deportiva y la precisión de percepción de competencia, se puede afirmar que ni los jóvenes que practican actividades físico-deportivas, ni los que no las practican, son precisos en la percepción de su competencia. Sin embargo, al comparar los dos grupos, resulta que los practicantes de actividades físico-deportivas son más precisos en sus valoraciones que los no practicantes. En cuanto a los no practicantes, como hemos advertido anteriormente, la falta de actividades físico-deportivas no permite que los niños tengan las experiencias fisico-deportivas necesarias para desarrollar su competencia percibida. La falta de instrucciones y feedbacks sobre su nivel de competencia motriz es un aspecto importante a tener en cuenta pues disponer de información sobre la ejecución de las tareas, además de influir en la creación de su competencia percibida, determina la precisión con la que el niño evaluara su nivel de habilidad (Goodway y Rudissill, 1997). Esto resulta de especial interés para los docentes quienes deberíamos recordar establecer la retroalimentación como elemento fundamental en el proceso de mejora del alumnado.

Finalmente, al analizar en qué medida el alumnado es preciso en su percepción de competencia, los datos muestran cómo quienes practican actividad físico-deportiva, sobrevaloran su competencia motriz. Tal y como expresaron Dunn y Watkinson (1994), una posible explicación a esta sobreestimación puede deberse al nivel de satisfacción que experimente el niño en dicha práctica. Estos autores, manifestaron, que, dependiendo de la satisfacción experimentada en una actividad fisico-deportiva concreta, la precisión con la que evalúan sus habilidades se ve influenciada, provocando una sobreestimación de su propia capacidad motora.

A modo de conclusión se pude determinar que el alumnado de educación secundaria mantiene que es competente, se percibe como tal y realiza práctica físico deportiva, y que quienes más competentes son, más competentes se perciben y más práctica realizan. Ello nos lleva a pensar, desde una perspectiva educativa, en la bondad de utilizar el Enfoque Temático de la Enseñanza de las Habilidades (ETEH) como modelo apropiado 
para la creación de hábitos físico-deportivos, que otorgando al alumnado del mayor número posible de experiencias y vivencias positivas, incida en el desarrollo la competencia motriz del alumnado, en la mejora de la competencia motriz percibida, que repercuta de manera positiva en la creación de hábitos de actividad física orientados al bienestar (Contreras, Arribas-Galarraga y Gutiérrez, 2017).

\section{Referencias}

Arruza Gabilondo, J.A., Arribas Galarraga, S., Otaegi Garmendia, O., González Rodríguez, O., Irazusta Adarraga, S. y Ruiz Pérez, L.M. (2011). Percepción de competencia, estado de ánimo y tolerancia al estrés en jóvenes deportistas de alto rendimiento. Anales de psicología, 27 (2), 536-543. Recuperado de: https://www.redalyc.org/pdf/167/16720051029.pdf

Barnett, A.L., Dawes, H. y Wilmut, K. (2012). Constraints and facilitators to participation in physical activity in teenagers with Developmental Coordination Disorder: an exploratory interview study. Child: care, health and development, 39 (3), 393-403. doi: 10.1111/j.13652214.2012.01376.x

Barnett, L.M., van Beurden, E., Morgan, P.J., Brooks, L.O. y Beard, J.R. (2009). Childhood Motor Skill Proficiency as a Predictor of Adolescent Physical Activity. Journal of Adolescent Health, 44, 252- 259. doi:10.1016/j.jadohealth.2008.07.004

Barnett, L.M., Morgan, P.J., van Beurden, E.V. y Beard, J.R. (2008). Perceived sports competence mediates the relationship between childhood motor skill proficiency and adolescent physical activity and fitness: a longitudinal assessment. International Journal of Behavioral Nutrition and Physical Activity, 40 (5), 1-12. doi:10.1186/1479-5868-540

Contreras-Jordan, O.R.; Arribas-Galarraga, S. y Gutiérrez, D. (2017). Didáctica de la educación física por modelos para la educación primaria. Editorial Sintesis. Madrid.

Harter, S. (1978). Effectance motivation reconsidered: Toward a developmental mode. Human Development, 21, 34-64. doi: $10.1159 / 000271574$

Dunn, J.L. y Watkinson, E.J. (1994). A study of the Relationship Between Physical awkwardness and Children's Perceptions of Physical 
Competence. Adapted Physical Activity Quarterly, 11, 275-283. doi: 10.1123/apaq.11.3.275

Eccles, J.S. y Wigfield, A. (2002). Motivational Beliefs, Values, and Goals. Annual Review of Psychology, 53, 109-132. doi: 0084-6570/02/02010109

García, E., Pérez, J.J., Rodríguez, P.L. y Moral, J.E. (2013). Relación de las capacidades coordinativas con la competencia motriz autopercibida en adolescentes. Trances, 5 (3), 213-228. Recuperado de: http://www.trances.es/papers/TCS\%2005_3_2.pdf

García, E., Rodríguez, P.L. y Pérez, J.J. (2013). Autopercepción de competencia motriz, práctica físico-deportiva federada y su relación con los niveles de actividad física habitual en escolares. Emás, Revista Digital de Educación Física, 4, (20), 1-20. Recuperado de: https:/ / dialnet.unirioja.es / servlet/articulo?codigo $=4196797$

Goodway, J.D. y Rudisill, M.E. (1997). Perceived Physical Competence and Actual Motor Skill Competence of African American Preschool Children. Adapted Physical Activity Quarterly, 14, 314-326. Recuperado de: https://pdfs.semanticscholar.org/275b/5d5910df36337a26f10421c0c 4c28af88ea5.pdf

Harter, S. (1988). Issues in the assessment of the self-concept of children and adolescents. En A. LaGreca (Ed.), Childhood assessment: Through the eyes of a child (pp.292-325). Boston: Allyn \& Bacon.

Haugen, T., Ommundsen, Y. y Seiler, S. (2013). The relationship between physical activity and physical self-esteem in adolescents: the role of physical fitness indices. Pediatric exercise science, 25, 138-153. doi: $10.1123 /$ pes.25.1.138

Horn, T.S. y Weiss, M.R. (1991). A developmental analysis of children's selfability judgments in the physical domain. Pediatric Exercise Science, 3, 310-326. doi: 10.1123/pes.3.4.310

Inchley, J., Kirby, J. y Currie, C. (2011). Longitudinal Changes in Physical Self-Perceptions and Associations With Physical Activity During Adolescence. Pediatric Exercise Science, 23, 237-249. Recuperado de: https://core.ac.uk/download/pdf/28974232.pdf

Khodaverdi, Z., Bahram, A., Khalaji, H. y Kazemnejad, A. (2013). Motor Skill Competence and Perceived Motor Competence: Which Best Precits 
Physical Activity among Girls? Iranian Journal of Public health, 42 (10), 1145-1150. Recuperado de: http://ijph.tums.ac.ir/index.php/ijph

Khodaverdi, Z., Bahram, A., Stodden, D. y Kazemnejad, A. (2016). The relationship between actual motor competence and physical activity in children: mediating roles of perceived motor competence and healthrelated physical fitness. Journal of sports sciences, 34(16), 1523-1529. doi: 10.1080/02640414.2015.1122202.

Lima, R. A., Pfeiffer, K., Larsen, L. R., Bugge, A., Moller, N. C., Anderson, L. B., \& Stodden, D. F. (2017). Physical activity and motor competence present a positive reciprocal longitudinal relationship across childhood and early adolescence. Journal of Physical activity and Health, 14(6), 440-447. doi: 10.1123/jpah.2016-0473

Luis-de Cos, I., Luis-de Cos, G. y Arribas-Galarraga, S. (2017). Práctica de actividad física y deporte, asociacionismo e intención de práctica futura en un grupo de adolescentes de 12 a 16 años. Sportis. Scientific Journal of School Sport, Physical Education and Psychomotricity, 3(3), 419-435. doi: 10.17979/sportis.2017.3.3.1899

Lopes, V.P., Rodrigues, L.P., Maia, J.A.R. y Malina, R.M. (2011). Motor Coordination as Predictor of Physical Activity in Childhood. Scandinavian Journal of Medicine and Science in Sport, 21(5), 663-669. doi: $10.1111 / \mathrm{j} .1600-0838.2009 .01$

McIntyre, F. (2009). A longitudinal Examination of the Contribution of Perceived Motor Competence and Actual Motor Competence to Physical Activity in 6 to 9 Year Old Children. (Tesis Doctoral). University of Notre Dame Australia: Frentmantle, WA.

Raudsepp, L. y Liblik, R. (2002). Relationship of perceived and actual motor competence in children. Perceptual and Motor Skills, 94, 1059-1070. doi: $10.2466 /$ pms.2002.94.3c.1059

Rigoli, D., Piek, J.P. y Kane, R. (2011). Motor Coordination and Psychosocial Correlates in a Normative Adolescent Sample. PEDIATRICS, 129 (4), 892-900. doi: $10.1542 /$ peds.2011-1237

Sporiš, G., Šiljeg, K., Mrgan, J. y Kević G. (2011). Self Evaluation of Motor and Functional Abilities among pupils. Croatina Journal of Education, $13,66-81$.

Stodden, D. F., Gao, Z., Goodway, J. D., \& Langendorfer, S. J. (2014). 
Dynamic relationships between motor skill competence and healthrelated fitness in youth. Pediatric Exercise Science, 26(3), 231241. doi:10.1123/pes.2013-0027

Stodden, D.F., Goodway, J.D., Langendorfer, S.J., Roberton, M.A., Rudisill, M.E., Garcia, C. \& Garcia, L.E. (2008). A Developmental Perspective on the Role of Motor Skill Competence in Physical Activity: An Emergent Relationship. Quest,60, 290-306. doi: 10.1080/00336297.2008.10483582

Stodden, D.F., Langendorfer, S.J. y Roberton, M.A. (2009). The Association Between Motor Skill Competence and Physical Fitness in Young Adults. Research Quarterly for Exercise and Sport, 80 (2), 223-229. doi: 10.1080/02701367.2009.10599556

Stodden, D. F., True, L., Langendorfer, S., \& Gao, Z. (2013). Associations among selected motor skills and health-related fitness: Indirect evidence for Seefeldt's proficiency barrier in young adults? Research Quarterly for Exercise and Sport, 84(3), 397-403. doi:10.1080/02701367.2013.814910

Urrutia-Gutierrez, S. (2014). La competencia motriz en el alumnado de la ESO y su relación con aspectos psicosociales (Tesis inédita de doctorado). Universidad del País Vasco, Donostia.

Vedul-Kjelsås, V., Sigmundsson, H., Stensdotter, A.-K. y Haga, M. (2012). The relationship between motor competence, physical fitness and selfperception in children. Child: care, health and development, 38 (3), 394-402. doi: 10.1111/j.1365-2214.2011.01275.x.

Viholainen, H., Aro, T., Purtsi, J., Tolvanen, A. y Cantell, M. (2013). Adolescents' school-related self-concept mediates motor skills and psychosocial well being. Britis Journal of Educational Psychology. doi: 10.1111/bjep. 12023

Wang, J., Liu, W. y Bian, W. (2013). Relationship between perceived and actual motor competence among college students. Perceptual and Motor Skills, 116, 272-279. doi: 10.2466/25.06.PMS.116.1.272-279

Washburn, R., \& Kolen, A. (2018). Children's Self-Perceived and Actual Motor Competence in Relation to Their Peers. Children, 5(6), 72. doi: 10.3390/children5060072.

Weiss, M.R. y Amorose, A.J. (2005). Children's Self-Perceptions in the Physical Domain: Between-and Within- Age Variability in Level, 
Gurutze Luis-de Cos, Saioa Urrutia-Gutierrez e Izaskun Luis-de Cos

Accuracy, and Sources of Perceived Competence. Journal of Sport \& Exercise Psychology, 27, 226-244. doi: 10.1123/jsep.27.2.226 